\title{
Guest Editorial Industrial Wireless Networks: Applications, Challenges, and Future Directions
}

W ITH THE rapid advance of wireless technologies, numerous emerging solutions and applications of industrial wireless systems have been developed. The present development of communication in industrial environments drives the need for ubiquitous access to distributed resources and services that are connected to things, devices, and systems. Service completions are typically perfected through smart APPS on wireless data delivery, such as $\mathrm{WiFi}$, Bluetooth, ZigBee, and 5G. The occurrence of Internet of Things (IoT) further catalyzes the advent of the wireless era. IoT aims at connecting all objects within the network and integrating them in a huge community. At this point, more and more emerging applications are under progress. However, the expansion and growth of wireless systems also inherit critical challenges. The challenges extend to many areas, key challenges include system reliability, leakage gas detection for public safety, security, energy efficiency, massive data gathering, energy harvesting long-range radio frequency identification (RFID) system, and robotic node localization. It is not uncommon that industrial environments are hostile to radio wave propagation, thus causing high uncertainties in data delivery. It is estimated that the huge population of wireless devices may reach seven trillion by 2020 [1]. Thus, it is necessary to enhance the current industrial wireless network and develop more advanced robust techniques to overcome the existing challenges.

Recently, numerous novel and prospective developments of industrial wireless network have been implemented on numerous applications such as healthcare, logistics, manufacturing process, home automation, and smart grid. For instance, in the development of healthcare, remote patient monitoring using ZigBee networks was proposed, which provided a real-time protection to the public, thus saving expenses by more than $\$ 45$ billion [1]. A new development on high-traffics smart metering supports the demand response application of smart grid [2]. Recent research demonstrates that the applications operate well in wireless systems and the offered services are alternated to be fully automated. The ultimate goal of the industrial applications is to provide the high-quality, efficient, safe, and sustainable living, and working environments to the public.

The aforementioned captioned that industrial wireless networks are facing various critical challenges [3]. These challenges mainly come from a large amount of connected devices with data transmission at extreme conditions such as dust, electromagnetic interference, and heat, thus causing uncertainty in industrial environment [4]. In [3], the major challenges of

Digital Object Identifier 10.1109/TII.2016.2528228 large-scale industrial wireless network are summarized as follows: safety, reliability, security, and energy consumption. Each challenge is a huge topic and sometimes the challenge may coexist with other challenges. Hence, it is observed that the comprehensive models addressing various challenges concurrently are more frequent to be found in the recent research studies.

The tremendous population growth of wireless devices will increase the loadings to the existing industrial systems. The enhancement of the wireless network performance is one of the future directions of industrial wireless networks. On the other hand, the ever increasing number of wireless sensors will facilitate the developments of various control schemes and applications. In particular, with the aid of diverse sensed data, full automation can be realized using broad control scheme. Gaining the insight from smart city development, fully automated wireless systems will be another key future direction.

Considering all these aspects, the Guest Editors have the pleasure to introduce the Special Session entitled "Industrial Wireless Networks-Applications, Challenges, and Future Directions" of the IEEE TRANSACTIONS ON INDUSTRIAL INFORMATICS. The selected six high-quality contributions cover the comprehensive solutions of wireless network developments, industrial applications, and wireless prototype designs.

The fundamental and important issue of system reliability in industrial environment was discussed and analyzed in the opening paper of this Special Section [5]. System reliability is one of the most critical issues in an industrial system in order to provide an efficient, safe, and productive operation. In [5], the generic heuristic algorithm of end-to-end reliability-aware scheduling (SchedEx) guarantees user-defined reliability and achieve scalable wireless sensor network (WSN) with small computation effort. Reliability is difficult to be evaluated or computed with exact value because the model normally requires a huge amount of data and deals with multiple uncertainties. It is the belief of the Guest Editors that guaranteeing reliability bounds is practical and important in industrial wireless environments, especially for large-scale projects. In some critical applications, reliability guarantee is ranked as the first priority. For instance, in healthcare application, a serious consequence will be resulted if the system reliability cannot reach a dedicated level. Besides, from the economy point of view, high reliability will demand less expenditure as a result of a stable production rate.

The second paper of the Special Session is contributed by a future explosive gas sensor design in which a wireless gas 
leak detection and localization solution was developed [6]. The development inspired a superior direction of the applications on industrial safety. In industrial environments, applications such as gas leakage detection and localization are crucial. An accurate identification of gas leakage as well as the faulty location will protect workers from risks. In addition, such a development reduces the expenditures of industrial injuries, thus rendering a huge contribution to the public safety. It is believed that massive data transmission handling of the sensors will be one of the key future research directions in industry wireless development.

The ever increasing number of wireless connections demands a serious consideration of security. In industrial environments, the introduction of environmental noises such as metallic frictions and engine vibrations renders the wireless interference and fading fluctuate drastically, resulting in a degradation of the secrecy capacity. This security-related issue has been addressed by the third paper of the Special Session [7]. In [7], an optimal sensor scheduling scheme has been developed to protect wireless transmission against the eavesdropping attack in a hostile industrial environment. The paper contributes a vision on modeling and enhancing security in wireless industrial environment with a full-scale analysis. Based on the analysis, key parameters such as electromagnetic interferences, multipath propagations, coexistence between different wireless protocols and quality of service should be considered in the future research direction.

Data gathering is an essential process for industrial WSN. The distributed battery-powered sensors transmit the stream of the sensed data to a sink node continuously, thus presenting a challenge on network lifetime. In essence, the sensor nodes close to the sink nodes normally consume much more energy as they are responsible to forward the data from other sensor nodes. In [8], the authors proposed an analytical model to estimate the entire network lifetime and determine the boundary of energy hole in a data-gathering WSN. The traffic load, energy consumption, and lifetime of sensor nodes during the entire network lifetime were estimated. The developed method was adopted in WSN routing for balancing the energy consumption and improving the network lifetime. This development contributes not only to the development of power-hungry industrial systems, but also benefits the deployment of smart wireless sensors in smart city developments.

In [9], an emerging solar self-powered sensor using RFID has been developed. The energy harvesting and unidirectional radiation pattern achieved the extension of RFID detection range. RFID applications are usually restricted by the extreme short-range communication. Conventional RFID tags perform tradeoff between communication range and energy consumption. Enhancing the RFID reader power can extend the detection range but such a large power may yield potential health hazard problem. In addition, increasing the RFID tag power demands a larger battery size, thus defeating the lightweight purpose of RFID tag. The contribution in [9] compromised the tradeoff practically. It will potentially facilitate a huge amount of applications, such as electronic toll collection systems, freight management, patient monitoring, and climate changes.

This Special Session closes with the paper of the node localization in robotic sensor network for pipeline inspection [10]. There are many important robotic applications but extremely useful applications can be found in dangerous and adverse environments, such as disaster scenes where human cannot or should not reach. The robotic applications improve the efficiency and safety of services. Besides, full automation is one of the ultimate goals in industrialized system, which aims at maximizing the production rate and minimizing the manpower and accidental injuries. It is envisaged that a large amount of robotic applications will be activated in the future industrial systems. As such, node localization contributed by [10] is essential for duty assignments and client recognitions. The node localization can also be implemented in other WSNs, in particular, for healthcare applications.

The Guest Editors of this Special Session would like to inspire the readers to gain insights for future industrial wireless network development. Such insights, in turn, facilitate readers' and researchers' creativity, vision, and knowledge. Finally, the Guest Editors would like to thank all the readers interested in this Special Session.

KIM FUNG TSANG, Guest Editor
City University of Hong Kong
Kowloon, Hong Kong
(e-mail: ee330015@ cityu.edu.hk)
MIKAEL GIDLUND, Guest Editor
Mid Sweden University
85170 Sundsvall, Sweden
(e-mail: mikael.gidlund@miun.se)
JoHAN ÅKERBERG, Guest Editor
ABB Corporate Research
72226 Västerås, Sweden
(e-mail: johan.akerberg@se.abb.com)

\section{ACKNOWLEDGMENT}

The Guest Editors would like to extend their sincere gratitude to all the authors who submitted their valuable contributions with great effort, and to all the reviewers who contributed their expertise and time for giving priceless reviews and advices for the submitted works. The Guest Editors believe that the selected contributions represent the current state of the art in the field and they will be of great interest to the industrial electronics community. Finally, they are grateful to the Editor-in-Chief of the IEEE TRANSACTIONS ON INDUSTRIAL INFORMATICS, Prof. K. F. Man, for offering the precious opportunity to organize this Special Section and for all the encouragement, support, and advices throughout the process. They would also like to thank S. McLain and L. Pattillo, TII staff, for their professional support and assistance during the whole preparation of this Special Section.

\section{References}

[1] H. Y. Tung, K. F. Tsang, H. C. Tung, K. T. Chui, and H. R. Chi, "The design of dual radio ZigBee homecare gateway for remote patient monitoring," IEEE Trans. Consum. Electron., vol. 59, no. 4, pp. 756-764, Nov. 2013.

[2] H. Y. Tung et al., "The generic design of a high-traffic advanced metering infrastructure using ZigBee," IEEE Trans. Ind. Informat., vol. 10, no. 1, pp. 836-844, Feb. 2014. 
[3] J. Åkerberg, M. Gidlund, and M. Bjorkman, "Future research challenges in wireless sensor and actuator networks targeting industrial automation," in Proc. 9th IEEE Int. Conf. Ind. Informat. (INDIN), Jul. 26-29, 2011, pp. 410-415.

[4] D. Yang et al., "Assignment of segmented slots enabling reliable real-time transmission in industrial wireless sensor networks," IEEE Ind. Electron., vol. 62, no. 6, pp. 3966-3977, Jun. 2015.

[5] F. Dobslaw, T. Zhang, and M. Gidlund, "End-to-end reliability-aware scheduling for wireless sensor networks," IEEE Trans. Ind. Informat., doi: 10.1109/TII.2014.2382335.

[6] F. Chraim, Y. Erol, and K. Pister, "Wireless gas leak detection and localization," IEEE Trans. Ind. Informat., doi: 10.1109/TII.2015.2397879.
[7] Y. Zou and G. Wang, "Intercept behavior analysis of industrial wireless sensor networks in the presence of eavesdropping attack," IEEE Trans. Ind. Informat., doi: 10.1109/TII.2015.2399691.

[8] J. Ren, Y. Zhang, K. Zhang, A. Liu, J. Chen, and X. Shen, "Lifetime and energy hole evolution analysis in data-gathering wireless sensor networks," IEEE Trans. Ind. Informat., doi: 10.1109/TII.2015.2411231.

[9] A. Abdulhadi and R. Abhari, "Multi-port UHF RFID tag antenna for enhanced energy harvesting of self-powered wireless sensors," IEEE Trans. Ind. Informat., doi: 10.1109/TII.2015.2470538.

[10] D. Wu, D. Chatzigeorgiou, K. Youcef-Toumi, and R. Ben-Mansour, "Node localization in robotic sensor networks for pipeline inspection," IEEE Trans. Ind. Informat., doi: 10.1109/TII.2015.2469636.

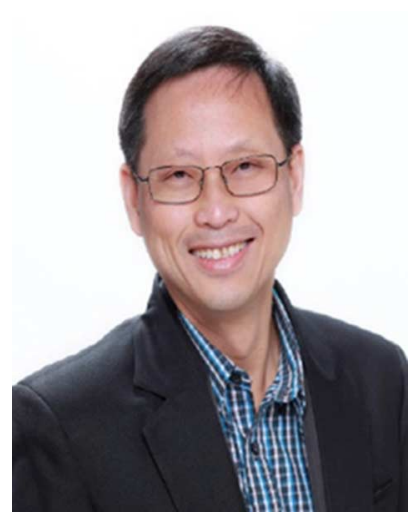

Kim Fung Tsang (M'95-SM'14) received the Ph.D. degree in microwave/millimeter wave engineering from the Cardiff University of Wales, Cardiff, U.K., in 1995.

$\mathrm{He}$ is currently an Associate Professor and the Director of the Wireless Sustainability Center with the Department of Electronic Engineering, City University of Hong Kong, Kowloon, Hong Kong. He has close ties with industry, and is working actively on radio frequency identification (ZigBee) for numerous applications, including energy management system for utilities, metering infrastructure, security, and office/home automation. He has authored about 180 technical papers.

Dr. Tsang is an Associate Editor and a Guest Editor of the IEEE TRANSACTIONS ON INDUSTRIAL INFORMATICS, the Chairman of the Technical Committee on Wireless and Cloud Architecture for Industrial Applications of the Industrial Electronics Society, Editor of the Korean Society for Internet Information Transactions on Internet and Information Systems, the ZigBee Chair of the IEEE Consumer Electronics Society (2014-2015), as well as the Chairman of the Internet of Things Special Users Group Hong Kong. He is a Fellow of the Hong Kong Institution of Engineers.
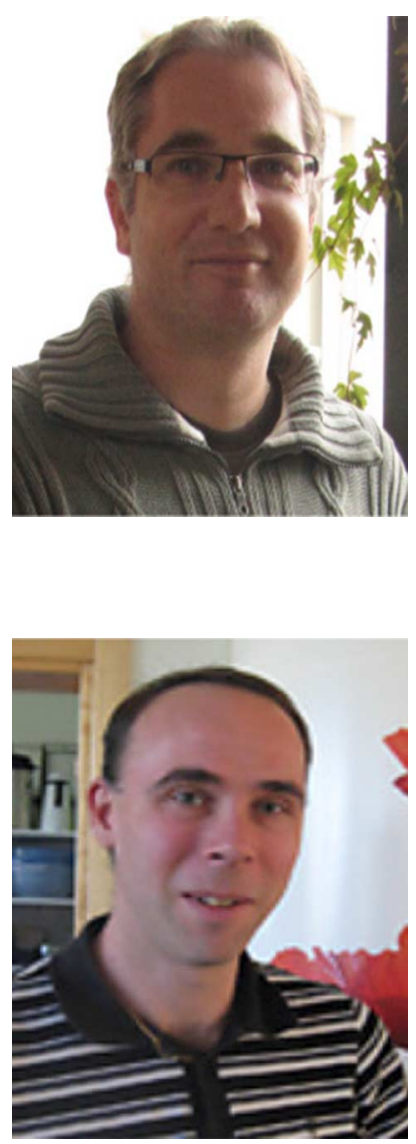

Mikael Gidlund (M'08) received the Lic.Eng. degree in radio communication systems from the Royal Institute of Technology (KTH), Stockholm, Sweden, and the Ph.D. degree in electrical engineering from Mid Sweden University, Sundsvall, Sweden, in 2004 and 2005, respectively.

Between 2006 and 2007, he was a Research Engineer and Project Manager at Acreo AB, Kista, Sweden. Between 2007 and 2008, he was a Project Manager and Senior Specialist in Wireless Systems at Nera Networks AS, Bergen, Norway. Between 2008 and 2013, he was a Senior Principal Scientist and Global Research Area Coordinator for Wireless Technologies at ABB Corporate Research, Västerås, Sweden. Since 2014, he has been a Full Professor of Computer Engineering with Mid Sweden University. He is also a Scientific Advisor with ABB Corporate Research. His research interests include wireless communication, signal processing, wireless sensor networks, and security, particularly for industrial applications.

Johan Åkerberg (M'08-SM'11) received the M.Sc. and Ph.D. degrees in computer science and engineering from Mälardalen University, Västerås, Sweden, 2007 and 2011, respectively.

$\mathrm{He}$ is a Principal Scientist and Global Research Area Coordinator for Embedded Systems and Electronics with ABB Corporate Research, Västerås. He has close to 20 years experience with $A B B$ in various positions such as Research and Development Project Manager, Industrial Communication Specialist, and Product Manager. $\mathrm{He}$ holds more than 10 patents (granted and pending applications) in the area of wired/wireless industrial automation, and is the author/co-author of numerous scientific publications in refereed conferences and journals. He is mainly working with communication for embedded real-time systems in industrial automation and is frequently invited to give talks to governmental bodies, international universities, and automation fairs.

Dr. Åkerberg organizes special sessions, holds tutorials, and acts as a Technical Program Committee Member for various distinguished Industrial Electronics Society conferences. 